\title{
Broad Spectral Domain Fluorescence Wavelength Modulation of Visible and Near-Infrared Emissive Polymersomes
}

P. Peter Ghoroghchian, ${ }^{* \dagger}$ Paul R. Frail,${ }^{\dagger}$ Kimihiro Susumu, ${ }^{\dagger}$ Tae-Hong Park, ${ }^{\dagger}$ Sophia P. Wu, ${ }^{\dagger}$ H. Tetsuo Uyeda, ${ }^{\dagger}$ Daniel A. Hammer, ${ }^{*, J}$ and Michael J. Therien ${ }^{\dagger, J}$

"School of Engineering and Applied Science, and Institute for Medicine and Engineering, University of Pennsylvania, 120 Hayden Hall, 3320 Smith Walk, Philadelphia, PA 19104, USA 'Department of Chemistry, University of Pennsylvania, 231 South 34th Street, Philadelphia, PA 19104, USA

hammer@seas.upenn.edu or therein@sas.upenn.edu

RECEIVED DATE ()

Tuning Vis- and NIR- Emissive Polymersomes

\section{Supporting Information Available:}

Materials- $\mathrm{PEO}_{30}-\mathrm{PBD}_{46}$ block copolymers under study were purchased from Polymer Source Inc, (Dorval, Quebec Canada). Compounds A-I, and variants with alternative meso-phenyl ancillary substituents, were synthesized following established cross-coupling methodology described elsewhere ${ }^{4}$.

\section{Methods-}

Vesicle Preparation: Formation of giant ( $>1 \mu \mathrm{m}$ diameter) and small $(<300 \mathrm{~nm}$ diameter) emissive polymersomes followed procedures described in ${ }^{3}$. Briefly, $100 \mu \mathrm{L}$ of $1 \mathrm{mM} \mathrm{PEO}_{30}-\mathrm{PBD}_{46}$ diblock copolymer solution and $5 \mu \mathrm{L}$ of $1 \mathrm{mM}$ solution of fluorophore species in methylene chloride were uniformly coated on the inside wall of a glass vial or on the surface of roughened Teflon, followed by evaporation of the solvent under vacuum for $>12 \mathrm{~h}$. Addition of aqueous solution (e.g. 250-300 mOsM sucrose, $290 \mathrm{mOsM}$ PBS, or DI water) and heating at $60{ }^{\circ} \mathrm{C}$ for $24 \mathrm{~h}$ lead to spontaneous budding of giant $(5-20 \mu \mathrm{m})$ emissive polymersomes (membrane loaded at $5 \mathrm{~mol} \%$ fluorophore:polymer) off of the glass (or Teflon) into the aqueous surroundings. Small $(<300 \mathrm{~nm}$ diameter) unilamellar polymersomes that possess appropriately narrow size distributions were prepared via procedures analogous to those used to formulate small lipid vesicles (sonication, freeze-thaw extraction, and extrusion).

Confocal microscopy: Fluorescence scanning confocal microscope images of giant $(5-20 \mu \mathrm{m}) \mathrm{NIR}-$ emissive polymersomes (internal solution: 290 mOsM sucrose; external solution: 290 mOsM PBS) were obtained using a Radiance 2000 Multi-photon Confocal System (Bio-Rad Laboratories, Hercules, CA) equipped with a $650 \mathrm{~nm}$ long-pass emission filter (excitation via argon laser, $\lambda_{\mathrm{ex}}=488 \mathrm{~nm}$ ). Micrographs were modified for brightness using Photoshop software (Adobe Systems, San Jose, CA).

Photophysical Studies: Aqueous solutions (DI water) of small, unilamellar emissive polymersomes ( $\mathrm{n}=10$ for each unique fluorophore:polymer formulation) were placed in $10 \mathrm{~mm}$ quartz optical cells and electronic absorption spectra for each of the membrane-incorporated fluorophore species were recorded on an OLIS UV/Vis/NIR spectrophotometry system that is based on the optics of a Cary 14 spectrophotometer (On-line Instrument Systems Inc, Bogart, GA). The solutions were then transferred 
to a glass vial, frozen in liquid $\mathrm{N}_{2}$, and lyophilized (FreeZone 4.5 L Benchtop Freeze Dry System, Labconco Corporation, Kansas City, MO; Model 77500) for 24 hours to destroy the vesicles and dry the polymer and fluorophore species. The dry samples were then taken up in the same volume of THF and their absorption spectra recorded. The concentrations of Compounds A-I, and variants with alternative meso-phenyl ancillary substituents, in the original polymersome solutions were calculated via a Beer's law determination using the absorption spectra taken in THF and the previously determined average molar extinction coefficients $\left(\varepsilon, \mathrm{M}^{-1} \mathrm{~cm}^{-1}\right)$ for each emitter in this solvent. These calculated concentrations, as well as the original NIRF absorption spectra in aqueous polymersome solutions, were utilized in order to determine the $\varepsilon$ for each polymersome-loaded fluorophore.

Fluorescence spectra of emissive polymersomes were obtained with a Spex Fluorolog-3 spectrophotometer (Jobin Yvon Inc, Edison, NJ) that utilized a dual S- and T-channel configuration and PMT/InGaAs/Extended-InGaAs detectors $\left(\lambda_{\text {ex }}=510 \mathrm{~nm}\right)$. Emission spectra were corrected using the spectral output of a calibrated light source supplied by the National Bureau of Standards.

Table S1: Spectral Properties of A-I as Observed in Tetrahydrofuran (THF) Solvent and in Aqueous Suspensions (DI water) of Polymersomes.

\begin{tabular}{|c|c|c|c|c|c|c|c|c|c|}
\hline \multirow{2}{*}{ Compound } & \multicolumn{4}{|c|}{ ABS THF } & \multicolumn{4}{|c|}{ ABS Aq. Polymersomes } & \multirow{2}{*}{$\begin{array}{c}\text { ABS Shifts } \\
\text { (Aq. Polymersomes - THF) } \\
\Delta v_{\max }\left[\mathrm{cm}^{-1}\right](\Delta \mathrm{E}[\mathrm{eV}])\end{array}$} \\
\hline & $\begin{array}{c}\lambda_{\max , \mathrm{Q}} \\
{[\mathrm{nm}]}\end{array}$ & \multicolumn{2}{|c|}{$\begin{array}{c}v_{\max } \\
{\left[\mathrm{cm}^{-1}\right]}\end{array}$} & $\log (\varepsilon)$ & $\begin{array}{c}\lambda_{\max , \mathrm{Q}} \\
{[\mathrm{nm}]}\end{array}$ & \multicolumn{2}{|c|}{$\begin{array}{c}v_{\max } \\
{\left[\mathrm{cm}^{-1}\right]}\end{array}$} & $\log (\varepsilon)^{*}$ & \\
\hline \multirow[t]{2}{*}{$\mathbf{G}$} & 570 & \multirow{2}{*}{\multicolumn{2}{|c|}{$\begin{array}{l}17,544 \\
16,340 \\
\end{array}$}} & 4.51 & 578 & \multirow{2}{*}{\multicolumn{2}{|c|}{$\begin{array}{l}17,301 \\
16,181\end{array}$}} & 4.46 & $-243(-0.03)$ \\
\hline & 612 & & & 4.47 & 618 & & & 4.53 & $-159(-0.02)$ \\
\hline \multirow[t]{2}{*}{$E$} & 572 & \multirow{2}{*}{\multicolumn{2}{|c|}{$\begin{array}{l}17,483 \\
15,576\end{array}$}} & 4.39 & 573 & \multirow{2}{*}{\multicolumn{2}{|c|}{$\begin{array}{l}17,452 \\
15,361\end{array}$}} & 4.36 & $-031(-0.00)$ \\
\hline & 642 & & & 4.81 & 651 & & & 4.82 & $-215(-0.03)$ \\
\hline \multirow[t]{2}{*}{ A } & 628 & \multirow{2}{*}{\multicolumn{2}{|c|}{$\begin{array}{l}15,924 \\
14,749\end{array}$}} & 4.60 & 632 & \multirow{2}{*}{\multicolumn{2}{|c|}{$\begin{array}{l}15,823 \\
14,451\end{array}$}} & 4.38 & $-101(-0.01)$ \\
\hline & 678 & & & 4.58 & 692 & & & 4.99 & $-298(-0.04)$ \\
\hline \multirow[t]{2}{*}{$\mathbf{H}$} & 568 & \multirow{2}{*}{\multicolumn{2}{|c|}{$\begin{array}{l}17,606 \\
16,340\end{array}$}} & 4.41 & 573 & \multirow{2}{*}{\multicolumn{2}{|c|}{$\begin{array}{l}17,452 \\
16,181\end{array}$}} & 4.40 & $-154(-0.02)$ \\
\hline & 612 & & & 4.33 & 618 & & & 4.43 & $-159(-0.02)$ \\
\hline \multirow[t]{2}{*}{$\mathbf{F}$} & 566 & \multirow{2}{*}{\multicolumn{2}{|c|}{$\begin{array}{l}17,668 \\
16,393\end{array}$}} & 4.46 & 572 & \multirow{2}{*}{\multicolumn{2}{|c|}{$\begin{array}{l}17,483 \\
16,181\end{array}$}} & 4.43 & $-185(-0.02)$ \\
\hline & 610 & & & 4.41 & 618 & & & 4.39 & $-212(-0.03)$ \\
\hline B & 695 & \multicolumn{2}{|c|}{14,388} & 4.71 & 710 & \multicolumn{2}{|c|}{14,085} & 4.83 & $-304(-0.04)$ \\
\hline \multirow[t]{2}{*}{ I } & 575 & \multirow{2}{*}{\multicolumn{2}{|c|}{$\begin{array}{l}17,391 \\
15,949\end{array}$}} & 4.65 & 578 & \multirow{2}{*}{\multicolumn{2}{|c|}{$\begin{array}{l}17,301 \\
15,823\end{array}$}} & 4.58 & $-090(-0.01)$ \\
\hline & 627 & & & 4.83 & 632 & & & 4.75 & $-126(-0.02)$ \\
\hline C & 717 & \multicolumn{2}{|c|}{13,947} & 4.47 & 727 & \multicolumn{2}{|c|}{13,755} & 4.50 & $-192(-0.02)$ \\
\hline \multirow[t]{2}{*}{ D } & 767 & & & 4.98 & 798 & & & 5.10 & $-506(-0.06)$ \\
\hline & & $\begin{array}{l}\text { lor } \\
\text { issi }\end{array}$ & $\mathrm{T}$ & ce & $\begin{array}{r}\text { Fluor } \\
\text { Aq. }\end{array}$ & $\begin{array}{l}\text { cen } \\
\text { oly }\end{array}$ & EI & $\begin{array}{l}\text { nission } \\
\text { mes }\end{array}$ & $\begin{array}{c}\text { Emission Shifts } \\
\text { (Aq. Polymersomes - THF) }\end{array}$ \\
\hline Compound & $\lambda_{\max , Q}$ & & & $\operatorname{ax}\left[\mathrm{cm}^{-1}\right]$ & $\lambda_{\max , Q}$ & & & $\operatorname{lax}\left[\mathrm{cm}^{-1}\right]$ & $\Delta v_{\max }\left[\mathrm{cm}^{-1}\right](\Delta \mathrm{E}[\mathrm{eV}])$ \\
\hline $\mathbf{G}$ & 616 & & & 16,234 & 622 & & & 16,077 & $-157(-0.02)$ \\
\hline & 674 & & & 14,837 & 680 & & & 14,706 & $-131(-0.02)$ \\
\hline $\mathbf{E}$ & 646 & & & 15,480 & 652 & & & 15,337 & $-142(-0.02)$ \\
\hline $\mathbf{A}$ & 685 & & & 14,599 & 697 & & & 14,347 & $-251(-0.03)$ \\
\hline $\mathbf{H}$ & 617 & & & 16,207 & 622 & & & 16,077 & $-130(-0.02)$ \\
\hline & 674 & & & 14,837 & 678 & & & 14,749 & $-088(-0.01)$ \\
\hline $\mathbf{F}$ & 652 & & & 15,337 & 663 & & & 15,083 & $-254(-0.03)$ \\
\hline B & 708 & & & 14,124 & 723 & & & 13,831 & $-293(-0.04)$ \\
\hline I & $\begin{array}{l}633 \\
687\end{array}$ & & & $\begin{array}{l}15,798 \\
14,556\end{array}$ & $\begin{array}{l}636 \\
682\end{array}$ & & & $\begin{array}{l}15,723 \\
14,663\end{array}$ & $\begin{array}{l}-075(-0.01) \\
+107(+0.01)\end{array}$ \\
\hline C & 757 & & & 13,210 & 757 & & & 13,210 & $0(0.00)$ \\
\hline D & 797 & & & 12,547 & 817 & & & 12,240 & $-307(-0.04)$ \\
\hline
\end{tabular}

*Absorbance $\varepsilon\left(\mathrm{M}^{-1} \mathrm{~cm}^{-1}\right)$ for each compound sequestered within hydrophobic polymersome membranes was calculated via a Beer's law determination (see experimental methods) and is reported on a per fluorophore basis. 
Supplemental Figure 1: Compounds A-I represent an extensive series of ethynyl- and butadiynylbridged bis- and tris[(porphinato)zinc] arrays that feature meso-to-meso $(\mathbf{A}, \mathbf{B}, \mathbf{C}, \mathbf{D})$, meso-to- $\beta(\mathbf{E}, \mathbf{F})$, and $\beta$-to- $\beta(\mathbf{G}, \mathbf{H}, \mathbf{I})$ linkage topologies between the (porphinato)zinc units. Scanning fluorescence confocal microscope images of giant $(5-20 \mu \mathrm{m})$ polymer vesicles feature compounds A-I loaded at 5 mol\% concentrations within their lamellar membranes (scale bar $=10 \mu \mathrm{m}$ ). Electronic absorption and fluorescence emission (inset) spectra are displayed for compounds A-I in THF (black) and aqueous solutions (DI water) of 100-nm-diameter emissive polymersomes (red).

Supplemental Figure 2: A. The extent to which the fluorophores are dispersed in membrane regions of differing dielectric properties impacts the Stokes shift of meso-to-meso ethynyl-bridged bis- and tris[(porphyrinato)zinc] arrays. Schematic of $(\alpha)$ the membrane/aqueous interface and $(\beta)$ the hydrophobic core of the bilayer membrane. B. Electronic absorption and fluorescence emission (inset) spectra of 3', 5'- aryl substituted ethynyl-bridged porphyrin trimers incorporated in differing membranous environments ( $\alpha-$ green vs. $\beta-$ red). 


\section{Supplemental Figure 1}
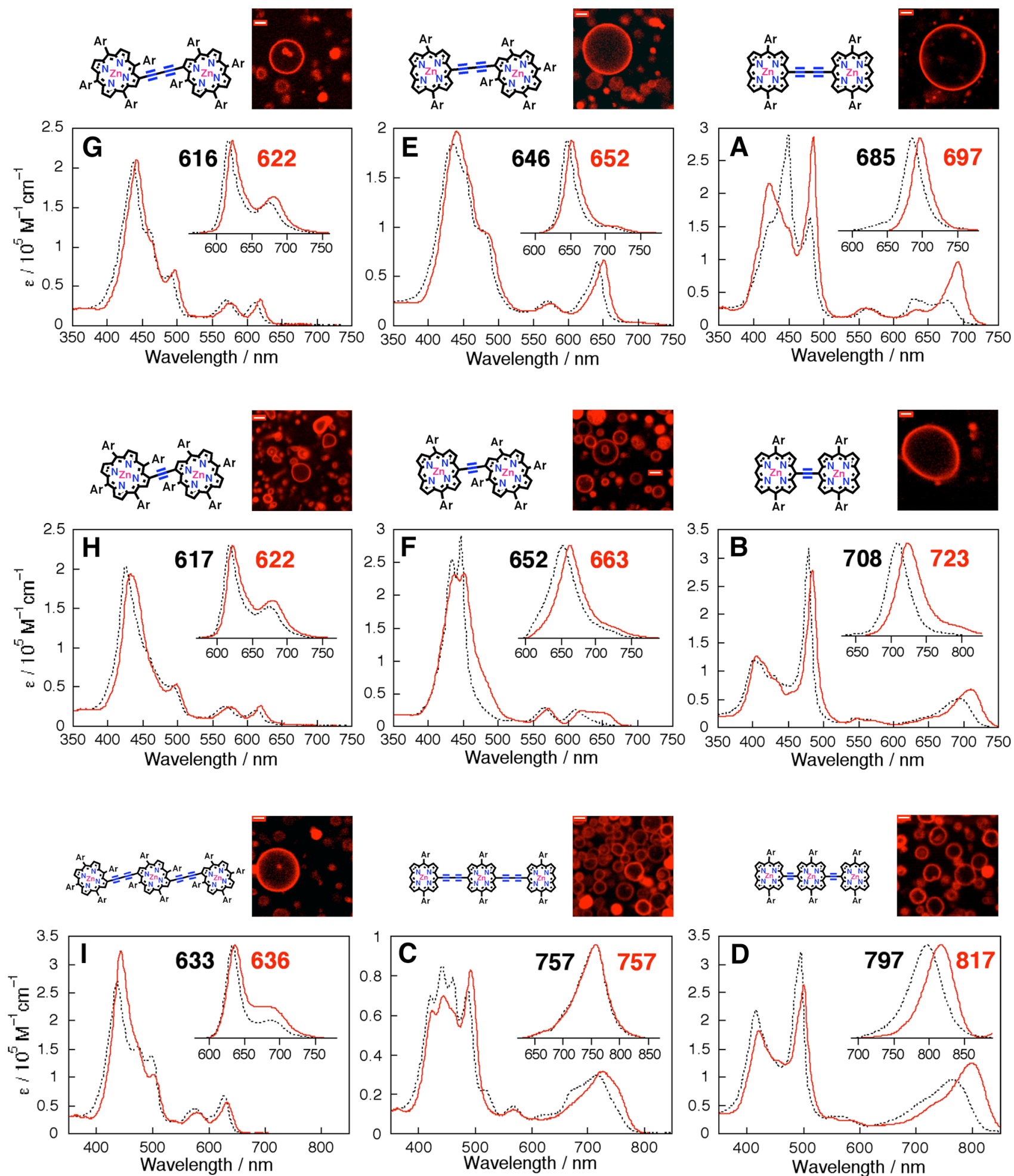
A

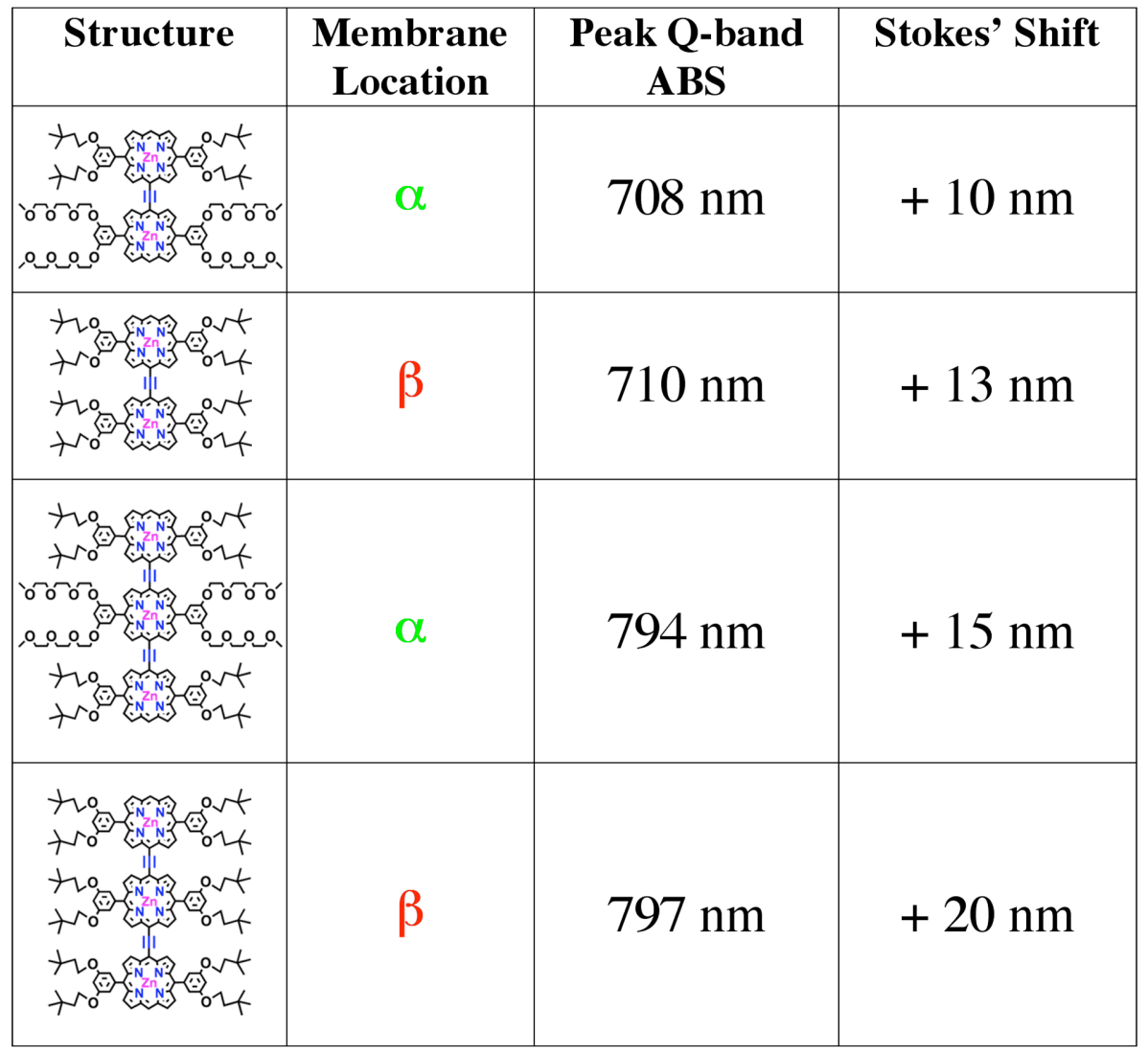

B
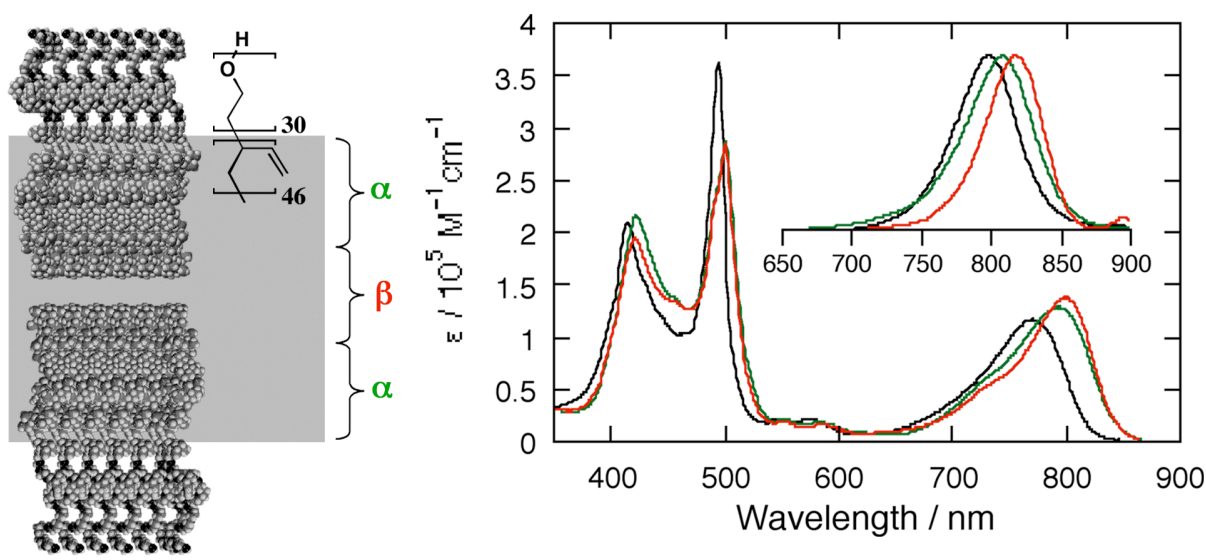